\title{
Sputtering at grazing ion incidence: Influence of adatom islands
}

\author{
Yudi Rosandi, ${ }^{1,2}$ Alex Redinger, ${ }^{3}$ Thomas Michely, ${ }^{3}$ and Herbert M. Urbassek ${ }^{1, *}$ \\ ${ }^{1}$ Fachbereich Physik und Forschungszentrum OPTIMAS, Universität Kaiserslautern, \\ Erwin-Schrödinger-Straße, D-67663 Kaiserslautern, Germany \\ ${ }^{2}$ Department of Physics, Universitas Padjadjaran, Jatinangor, Sumedang 45363, Indonesia \\ ${ }^{3} I I$. Physikalisches Institut, Universität Köln, Zülpicherstraße 77, D-50937 Köln, Germany
}

(Received 12 March 2010; revised manuscript received 10 August 2010; published 22 September 2010)

\begin{abstract}
When energetic ions impinge at grazing incidence onto an atomically flat terrace, they will not sputter. However, when adatom islands (containing $N$ atoms) are deposited on the surface, they induce sputtering. We investigate this effect for the specific case of $83^{\circ}$-incident $5 \mathrm{keV}$ Ar ions on a $\mathrm{Pt}$ (111) surface by means of molecular-dynamics simulation and experiment. We find that - for constant coverage $\Theta$ - the sputter yield has a maximum at island sizes of $N \cong 10-20$. A detailed picture explaining the decline of the sputter yield toward larger and smaller island sizes is worked out. Our simulation results are compared with dedicated sputtering experiments, in which a coverage of $\Theta=0.09$ of Pt adatoms are deposited onto the Pt (111) surface and form islands with a broad distribution around a most probable size of $N \cong 20$.
\end{abstract}

DOI: 10.1103/PhysRevB.82.125440

PACS number(s): 79.20.Rf, 79.20.Ap, 61.80.Lj

\section{INTRODUCTION}

Energetic ion incidence on surfaces is responsible for surface erosion and damage production. However, at grazing incidence angles these effects are strongly reduced. Flat surfaces reflect ions at glancing incidence with only little energy input into the surface. However, even slight imperfections of the surface may alter the situation dramatically. The effect of surface steps has been analyzed in detail. ${ }^{1-4}$ It was shown that if the ion impact point is in the vicinity of an ascending step edge, an ion may sputter as many atoms as for normal incidence. The sputter yield of a stepped surface is thus primarily governed by the step density. Similarly, the influence of single adatoms on a surface was analyzed and it was found that for small coverages, the sputter yield is linear in the adatom coverage. ${ }^{5-7}$

Real surfaces may be covered by adatom (and vacancy) islands as well, and the question arises how the sputter yield is influenced by these islands. One might think that adatom islands simply interpolate between the effects of isolated adatoms and straight step edges. However, this view is too simple, and a detailed picture of the effect of adatom islands on sputtering will be developed here.

Grazing-ion incidence on surfaces is interesting from a fundamental point of view but it also finds applications. Here, we mention, in particular, their ability to pattern surfaces, and to induce ripple structures on the surface. ${ }^{8}$ Recently, the application of glancing-ion incidence on nanopatterning of surfaces has been investigated and the detailed atomistic aspects of glancing-ion incidence on surfaces were shown to be relevant for an understanding of the initial stages of pattern formation on metallic surfaces. ${ }^{2,9,10}$

The effect of surface defects on the trajectories of incident ions has already been considered in the 1970s. Interest in this question arose due to the technique of low-energy ion scattering which was used successfully to study the composition and structure of the surface. ${ }^{11-14}$ It allowed to measure preexisting surface defects and also adsorbates. ${ }^{15-18}$ These experiments could be interpreted using simulations based on the binary-collision approximation, in which the result of the ion impact on the target could be ignored. ${ }^{11,12}$ These simulations did not allow to determine sputtering or ion-induced damage. Therefore, in our present analysis of the effect of surface defects on sputtering, we have to go beyond the binary-collision approximation and to employ full moleculardynamics simulations.

In this paper we approach the question as to how adatom islands contribute to sputtering at grazing incidence by experiment and simulation. Using molecular-dynamics simulation we can address this question systematically, at least for small island sizes, and obtain a detailed atomistic view of the sputter processes in dependence of the ion impact point. These simulations are performed for $5 \mathrm{keV} \mathrm{Ar}$ impact on the Pt (111) surface at an incidence angle of $83^{\circ}$ to the normal. At this angle, a flat terrace reflects the ion beam without being damaged or sputtered, and hence all damage can be attributed unambiguously to the adislands at the surface. We note that we investigated previously the effects of a varied incidence angle on sputtering from surface steps and found qualitatively similar results. For the $5 \mathrm{keV}$ Ar impact at $83^{\circ}$ incidence onto the Pt (111) surface, where already a considerable body of information on the sputtering behavior both from experiment and simulation is available,${ }^{1-7}$ we perform dedicated experiments. Here, the Pt surface is initially covered by Pt adatom islands which have been deposited on the surface prior to ion bombardment. Simulations and experiment agree satisfactorily, when the size distribution of the adatoms is taken into account.

\section{METHOD}

\section{A. Simulations}

We consider the impact of $5 \mathrm{keV}$ Ar atoms on a Pt (111) surface at a fixed incidence angle of $83^{\circ}$ toward the surface normal. The incidence azimuth is chosen such that its projection onto the surface is aligned in the [1 $\overline{1} 2]$ direction. Our simulation crystallite contains 15 layers; each layer extends 


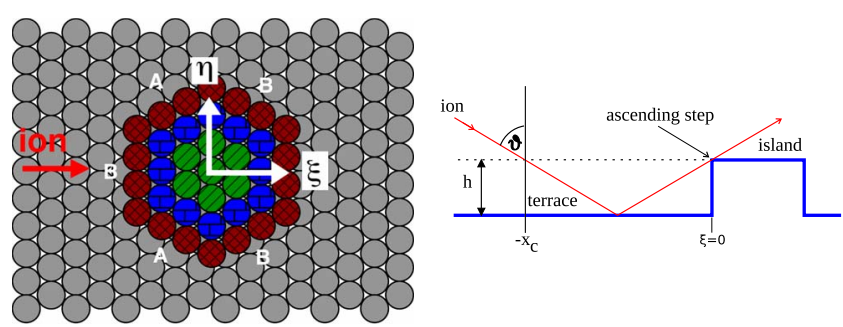

FIG. 1. (Color online) (a) Sketch of the configuration of adatom islands on top of the Pt (111) surface. The concentric rings display 7-, 19-, and 37-islands. Ions impinge from the left-hand side, along the [1 $\overline{1} 2]$ azimuth (red arrow at the left-hand side). A and B steps bounding the island are indicated. The $(\xi, \eta)$ coordinate system on top of the surface is indicated; its origin is at the center of the ascending step edge of the island under consideration (here the $N$ $=1$ adatom). (b) Schematical side view of an ion trajectory hitting the island after reflection from the terrace (so-called indirect hit). The ascending edge of the island is positioned at $\xi=0$; the coordinate $\xi$ measures the ion impact position at the height of the island (dotted line). The critical distance $x_{c}$, Eq. (7), is-for a straight-line ion trajectory-the largest distance of the ion impact to the step edge which lets the ion collide with the island.

$100 \AA$ in the direction of the ion beam, and is $87 \AA$ broad. Thus the total number of Pt atoms in the crystallite amounts to 20160 . We employ a many-body interaction potential ${ }^{19}$ for the Pt-Pt interaction while the Ar projectile interacts via the purely repulsive Ziegler-Biersack-Littmark (ZBL) potential ${ }^{20}$ with Pt. The simulation is performed at a temperature of $0 \mathrm{~K}$ by relaxing the target structure to minimum potential energy and quenching the kinetic energy. The interatomic interaction potential has been employed previously for sputtering simulations, and showed fair agreement with experiment, both for normal and glancing ion incidence angles. ${ }^{3,19}$

Before the start of the simulation, we put adatom islands containing $N$ adatoms on the surface. In this investigation, we choose sizes of $N=7,19,37$; in these special cases, the island forms a regular hexagon on the (111) surface. The cases of a single isolated adatom $(N=1)$ and of a densepacked surface step (corresponding to $N=\infty$ ) have been simulated previously. ${ }^{1-4,6,10,21}$ The adatoms are put on stable fcc sites on top of the crystallite. The island position, as well as that of all surface atoms are relaxed. The height of the adatoms above the surface is $h=2.26 \AA$. The [nearestneighbor (NN)] distance between adatoms is $r_{\mathrm{NN}}=2.77 \AA$.

The Ar ion impact direction-along the [1̄12] azimuthhas been chosen such that for a central impact, the ion encounters a dense-packed step [compare Fig. 1(a)] as the ascending step of the island. Each trajectory is followed for 10 ps. This time is sufficient to decide on the fate on the projectile and the adatom, and also to determine the sputter yield reliably. The damage production on the surface, however, may still change after this time due to relaxation and diffusion processes. We are confident, though, that the qualitative features of the damage production may be analyzed already at this time.

We consider all those atoms as sputtered that have zeropotential interaction energy with the target; due to our cutoff radius of $5.1 \AA$ this means that they are a distance of at least
$5.1 \AA$ away from all substrate atoms or adatoms of the target. The damage production will be quantified in the following by counting all those atoms as adatoms, which are above the initial substrate surface; the initial adatoms are included in the count.

\section{B. Sputter yield}

Molecular-dynamics simulation calculates the sputter yield of a surface $A$ as the average

$$
\bar{Y}=\frac{1}{A} \iint Y(\boldsymbol{\rho}) d^{2} \rho .
$$

Here, $\boldsymbol{\rho}$ is the impact point of the ion on the surface and $Y(\boldsymbol{\rho})$ is the corresponding sputter yield. At a temperature of $0 \mathrm{~K}$, $Y(\boldsymbol{\rho})$ is a deterministic function, which is calculated by performing a molecular-dynamics simulation for each value of $\boldsymbol{\rho}$.

In our case of interest, the sputter yield of an ion hitting a flat terrace vanishes; only ions hitting an adatom island contribute to sputtering. Let us consider the special case that exactly one adisland consisting of $N$ adatoms is situated in $A$. We introduce the sputter cross section of the adisland as

$$
\sigma_{\mathrm{sp}}^{N}=\iint Y(\boldsymbol{\rho}) d^{2} \rho,
$$

such that

$$
\bar{Y}=\frac{\sigma_{\mathrm{sp}}^{N}}{A} .
$$

This terminology is analogous to that used for sputtering of an adsorbate- or adatom-covered surface. ${ }^{7,22,23}$ This particular situation can be related to the case where several adislands are situated on the surface by the following argument. If one $\mathrm{N}$-adisland is on the surface, the nominal coverage is

$$
\Theta=\frac{N A_{0}}{A},
$$

where $A_{0}=6.67 \AA^{2}$ is the area an atom covers on the surface. For small coverages $\Theta \ll 1$, where the effect of individual islands does not interfere with each other, it may be assumed that $\bar{Y}$ is proportional to the coverage $\Theta$, and we may define a sputter coefficient $y_{N}$ via

$$
\bar{Y}=y_{N} \Theta .
$$

The sputter coefficient $y_{N}$ is thus calculated from simulation via

$$
y_{N}=\frac{\sigma_{\mathrm{sp}}^{N}}{N A_{0}} .
$$

We shall use $y_{N}$ as our central simulational result, which allows to compare to the experimentally accessible sputter yield, $\bar{Y}$, via Eq. (5).

\section{Experimental}

The experiments have been performed in a variabletemperature scanning tunnelling microscopy (STM) appara- 

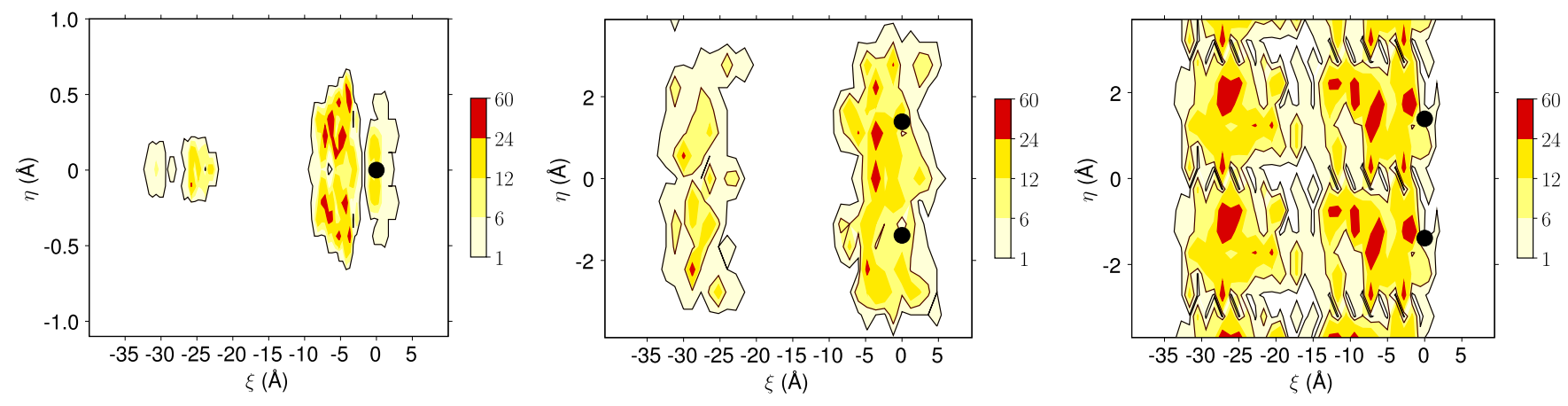

FIG. 2. (Color online) Contour plot of sputter yield $Y(\xi, \eta)$ for various adatom islands on the Pt (111) surface. The incoming ion flight direction is along the $\xi$ axis. Black dots mark the positions of the island edge atoms facing the ion beam, positioned at $\xi=0$. Note the difference between the $\xi$ and the $\eta$ scale, and in the $\eta$ scales between subfigures (a), (b), and (c). (a) isolated adatom, $N=1$, Ref. 6. (b) Adatom island, $N=7$. (c) Step, $N=\infty$.

tus with a base pressure in the $10^{-11}$ mbar range. Sample cleaning was accomplished by cycles of ion erosion with 5 $\mathrm{keV} \mathrm{Ar}^{+}$ions and flash annealing to $1270 \mathrm{~K}$. Deposition of platinum is accomplished by thermal evaporation out of a platinum wire which is wrapped around a tungsten wire to ensure mechanical stability. The evaporation rate in the experiments was set to $5 \times 10^{-3} \mathrm{ML} / \mathrm{s}$, where $1 \mathrm{ML}$ (monolayer) corresponds to the areal density of the $\mathrm{Pt}(111)$ substrate of $1 / A_{0}=1.50 \times 10^{19}$ atoms $/ \mathrm{m}^{2}$.

Ion bombardment was performed with a differentially pumped ion source providing a mass separated $5 \mathrm{keV} \mathrm{Ar}^{+}$ ion beam. The ions impinge under an angle of $\vartheta=83^{\circ}$ with respect to the surface normal along the [1피 azimuth. The ion flux was calibrated by a movable Faraday cup at the sample location and was set to $1.8 \times 10^{16} \mathrm{~m}^{-2} \mathrm{~s}^{-1}$. During ion exposure the sample temperature was set to $155 \mathrm{~K}$ and the ion fluence used was 0.5 MLE. 1 MLE (monolayer equivalent) corresponds to the areal density of the Pt (111) substrate.

STM imaging was performed at the temperature of the experiment or below to prevent thermal healing of the structures formed.

\section{SIMULATION RESULTS}

\section{A. Result for the $N=7$ island}

We start with the analysis of sputtering induced by a specific island, $N=7$. Its form is indicated in Fig. 1(a). As indicated in Fig. 1(a), the edge of the hexagonal island is composed of two types of dense-packed step edges. B steps are $\{111\}$ microfacets while A steps are $\{100\}$ microfacets. The ion is incident from the left along the [1-12] azimuth. It hits either perpendicularly onto a B step of width $r_{\mathrm{NN}}$, or onto an A step which is at an angle of $60^{\circ}$ to the ion beam direction above and below the B step. The total sputter cross section obtained from our simulation amounts to $1147 \AA^{2}$. This value may be compared with that of a single adatom, $110 \AA^{2}$ (Ref. 6) and we observe that the total effect of a 7-island is larger than that of 7 isolated adatoms.

In Fig. 2(b) we display a contour plot of the sputter yield $Y(\boldsymbol{\rho})$ for sputtering off a 7 -island; it is contrasted with the corresponding plots for sputtering off a single adatom [Fig. 2(a)] and an infinite step [Fig. 2(c)]. We introduced a coordinate system $\boldsymbol{\rho}=(\xi, \eta)$ on the surface, where the $\xi$-axis points along the ion flight direction, i.e., into the [1- 12$]$ azimuth [compare Fig. 1(a)]. The adatom island is positioned such that the center of its ascending edge is at $(\xi=0, \eta$ $=0)$. The ion impact point is measured at the height of the adisland atoms, not of the terrace.

We observe that only ions hitting in front of the island induce sputtering; ions hitting on the upper terrace behind the step or on the island will be reflected without putting much energy into the surface. Furthermore, two distinct areas in front of the island can be identified which contribute to sputtering: A zone which is immediately adjacent to the island and extends $12 \AA$ in front of it (for the 7-island); this is the direct-hit zone in which the ion collides violently with the edge of the island and induces sputtering. The second zone extends 19-35 $\AA$ in front of the step, and is called the indirect-hit zone; here the ion hits the flat terrace in front of the island, is reflected and then hits the step edge, inducing sputtering. Ions in between these two zones pass under the island without transferring energy to it. For the case of an extended step, it has been shown that these ions are caught in channels immediately below the upper terrace; upon dechanneling they may induce damage and sputtering but this only happens at a long distance behind the step edge (at least $x_{c} / 2=19 \AA$, cf. the discussion below, but extending to more than $100 \AA$ ).

A simple geometric model has been set up to relate the extension of these zones to the step height $h$ and the ion incidence angle $\vartheta$ with respect to the surface normal, cf. Fig. 1(b). ${ }^{1}$ The critical distance

$$
x_{c}=2 h \tan \vartheta
$$

quantifies the largest distance from the step edge, at which an ion-after reflection from the lower terrace at $x_{c} / 2-$ will hit the step edge. $x_{c}$ terminates the indirect-hit zone and thus the zone of influence. Ions impinging at $x_{c} / 2$ pass under the step edge atom; this characterizes the passing zone. In our case, it is $x_{c}=38.8 \AA$.

The influence of the island extends laterally through a width of around $7.5 \AA$, compared to the case of the single 


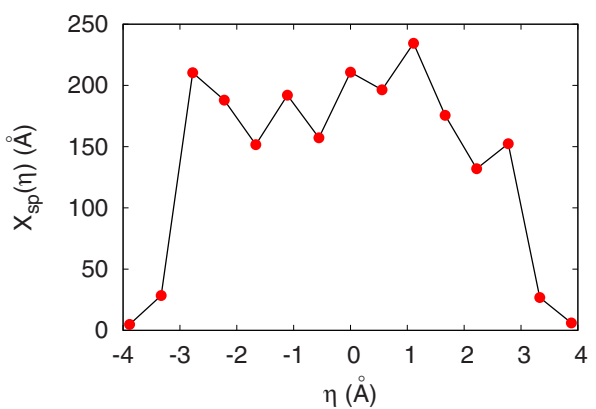

FIG. 3. (Color online) Dependence of sputter length $X_{\mathrm{sp}}$ on lateral position $\eta$ of ion impact on 7 -island. Lines to guide the eyes.

adatom, where the influence extends only over $1.3 \AA$. For a further discussion, it is useful to introduce the integral

$$
X_{\mathrm{sp}}(\eta)=\int Y(\xi, \eta) d \xi,
$$

which we call the sputter length. We can use it to quantify the lateral dependence of the sputter yield. Figure 3 shows that - apart from fluctuations - the sputter length is rather constant over the full width of the island and only decays for the last $1 \AA$ rather rapidly to 0 . This constancy already elucidates from Fig. 2(b). We shall use this constancy as it allows us to extrapolate our simulation results for central impact $\eta=0$ to a discussion of the entire island sputter behavior.

\section{B. Dependence on island size $N$}

In view of the independence of the sputter behavior on the lateral position of the ion impact point established for 7-islands, we discuss the $N$ dependence of sputtering with the help of the sputtering yields for central impact. Here we average data over the central part $\left(-r_{\mathrm{NN}} / 2 \leq \eta \leq+r_{\mathrm{NN}} / 2\right)$ of the island and calculate the sputter yield in the stripe

$$
\tilde{Y}(\xi, 0)=\frac{1}{r_{\mathrm{NN}}} \int_{-r_{\mathrm{NN}} / 2}^{+r_{\mathrm{NN}} / 2} Y(\xi, \eta) \mathrm{d} \xi .
$$

The sputter yields are displayed in Fig. 4 for island sizes of $N=7,19,37$, and compared to the case of a single adatom

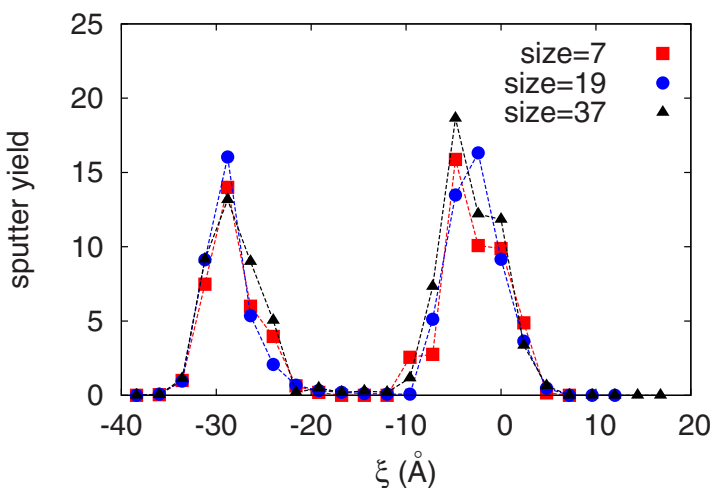

and an extended step. The central sputtering yields for the three islands studied are remarkably similar. The three regions discussed above (direct-hit, passing, and indirect-hit zone) have similar extensions and give rise to similar sputtering yields. In comparison, the single adatom has a considerably smaller yield. In particular, the indirect-hit zone has shrunk in length and also contributes less to sputtering. As shown in the contour plot, Fig. 2, this is, in particular, due to a smaller lateral extension of the indirect-hit zone; it is easy for the ion to miss the single adatom after reflection of the terrace. The extended step edge, however, shows a rather changed sputter characteristics: The sputter contributions of both the direct- and the indirect-hit zone have increased quantitatively, and also the passing zone now contributes to sputtering. The latter phenomenon has been ascribed above to the fact that the ion can no longer pass below a semiinfinite upper terrace but will at some time be dechanneled and then may contribute to sputtering. From the geometric model outlined above, we argue that if the length of the island is $x_{c} / 2$ or larger, an ion passing under the island may hit the step edge atom on the far side of the island; this corresponds to island sizes of $N \geq 61$. However, from our previous work with sputtering from step edges we know that the ion is channeled between the island atoms (upper terrace) and the lower terrace; hence larger islands are needed in order to fill up the passing-zone dip visible for small islands $(N \leq 39)$ of Fig. 4(a).

\section{Sputter cross section and sputter coefficient}

Figure 5 displays the sputter length $X_{\mathrm{sp}}=X_{\mathrm{sp}}(0)$ introduced in Eq. (8) and shows that it is rather constant for the islands studied here $(N=7,19,37)$. In agreement with the data shown in Fig. 4, the single adatom has a by a factor of $1 / 3$ decreased sputter length while the step edge is more productive by a factor of 2 , cf. also Table I.

These data can be used to approximate the sputter cross section of Eq. (2) as

$$
\sigma_{\text {sp }}^{N}=X_{\text {sp }}(0) L_{\perp},
$$

where $L_{\perp}$ denotes the width of the island perpendicular to the ion beam. The sputter coefficient then is

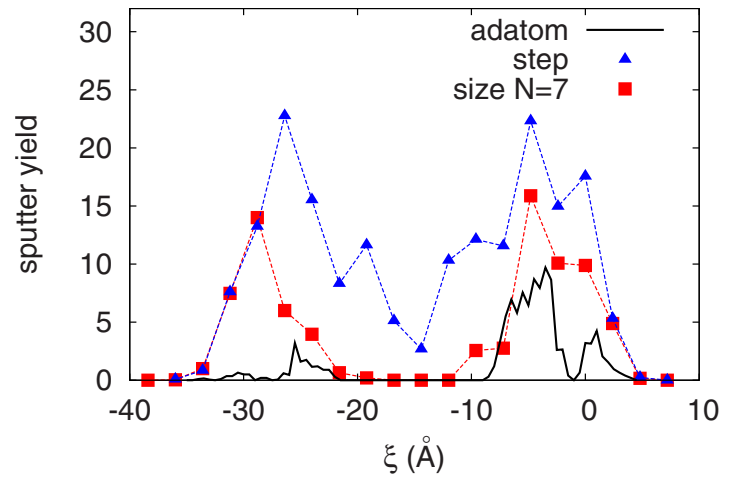

FIG. 4. (Color online) Dependence of the sputter yield on the distance $\xi$ to the ascending island edge for central impacts, averaged over a stripe of width $r_{\mathrm{NN}}$ around $\eta=0$, Eq. (9). Note that the data for the single adatom, $N=1$, and for the step, $N=\infty$, are identical to the fully laterally averaged sputter yields. (a) Island size $N=7,19,37$. (b) Island size $N=1,7, \infty$. Lines to guide the eyes. 


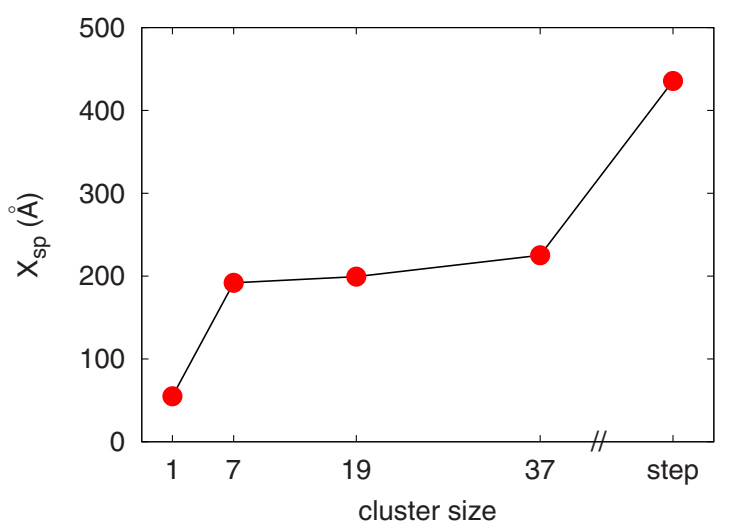

FIG. 5. (Color online) Dependence of the sputter length $X_{\mathrm{sp}}(0)$ on the island size $N$. Data calculated for $\eta=0$. Lines to guide the eyes.

$$
y_{N}=\frac{X_{\mathrm{sp}}(0) L_{\perp}}{N A_{0}} .
$$

The width which the island exposes to the ion can be calculated from a geometric argument: we set $L_{\perp}$ equal to the diameter of a circle of equal area as the hexagon

$$
L_{\perp}=2 \sqrt{\frac{A}{\pi}}=\sqrt{\frac{2 \sqrt{3}}{\pi}} \sqrt{N} r_{\mathrm{NN}}=1.05 \sqrt{N} r_{\mathrm{NN}} .
$$

This is obviously an approximation but features the essential $\sqrt{N}$ dependence. Inserting this expression in Eq. (11) we obtain

$$
y_{N}=1.21 \frac{X_{\mathrm{sp}}(0)}{r_{\mathrm{NN}}} \frac{1}{\sqrt{N}} \cong \frac{100}{\sqrt{N}} .
$$

For the latter approximation, we have used the sputter length of 37-islands, $225.7 \AA$, cf. Table I.

Figure 6 shows our simulation results for the sputter coefficient for island sizes $N \leq 37$ together with a plot of Eq. (13). This is the central result of the simulation. In addition to the simulated data for $N=1$ and $N=7$, the figure contains data based on ion impacts along the central line, $X_{\mathrm{sp}}(0)$, for $N=19$ and 37; in order to calculate the full sputter yield for the latter data, we made use of Eq. (11), i.e., multiplied with the island width, $L_{\perp}$. Finally, for $N>37$, we did not employ any further simulation data but simply assumed the sputter

TABLE I. Sputter length, $X_{\mathrm{sp}}(0)$, Eq. (8), and sputter coefficient $y_{N}$ for adatom islands of size $N$. $N=\infty$ corresponds to an extended B step.

\begin{tabular}{ccc}
\hline \hline \multirow{2}{*}{$N$} & $\begin{array}{c}X_{\mathrm{sp}} \\
(\AA)\end{array}$ & \multicolumn{1}{c}{$y_{N}$} \\
\hline 1 & 55.0 & 16.5 \\
7 & 190.8 & 22.6 \\
19 & 200.0 & 17.5 \\
37 & 225.7 & 15.2 \\
$\infty$ & 435.5 & n.a. \\
\hline \hline
\end{tabular}

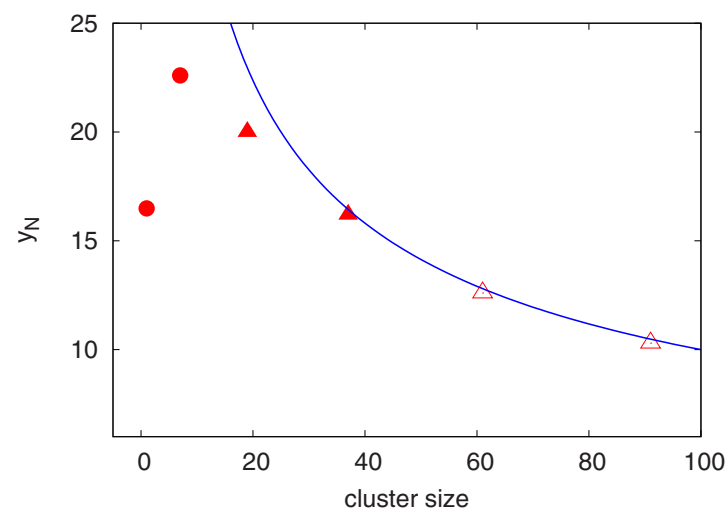

FIG. 6. (Color online) Sputter coefficient $y_{N}$ vs island size $N$. Full circles: simulation results. Full triangles: Eq. (11), using simulation results for the sputter length $X_{\mathrm{sp}}(0)$. Open triangles: Eq. (11), using $X_{\mathrm{sp}}(0)$ of the 37-island. Line: analytical law, Eq. (13), valid for $N \gtrsim 20$.

yield to increase with the exposed width of the island, $L_{\perp}$ while keeping $X_{\text {sp }}(0)$ fixed to its value as calculated for $N$ $=37$, cf. Fig. 3 .

The data demonstrate that our approximation [Eq. (13)] describes our simulation results satisfactorily for $N \gtrsim 20$. For smaller island sizes, the sputter coefficient decreases since the sputter length decreases. Thus it turns out that islands of size $N \cong 10-20$ are most effective for sputtering; for larger sizes, the interior and back side of the island do not contribute to sputtering, and for smaller sizes, the reduction in particular of the indirect hit zone decreases sputtering.

\section{Azimuth dependence}

All the above results have been derived for an impact azimuth of [1-12]. From previous work on the sputtering of B-step edges, it is known that there exists a strong azimuth dependence of sputter yields. In simulation, for an impact azimuth of $[1 \overline{1} 0]$, sputter yields decrease by roughly $50 \%$ in simulation compared to the [1-12] azimuth; in experiment the reduction amounts to $40 \% .^{3}$ This strong reduction has been attributed mainly to the fact that the (111) terrace appears more corrugated for an ion incident along the [1 $\overline{10} 0]$ azimuth. We include this reduction in sputter yield for the $[1 \overline{1} 0]$ azimuth in our calculation of the sputter coefficient by reducing the result of Eq. (13) by 50\%:

$$
y_{N} \cong \frac{50}{\sqrt{N}} .
$$

We proceed to present a more detailed discussion of the azimuth dependence. To this end, we display in Fig. 7 our results for sputtering of an $N=7$ island for the [1 $\overline{10}$ ] azimuth and compare it to those obtained above for the [112] azimuth. Note that for the [1피 azimuth, no part of the step edge is perpendicular to the ion incidence azimuth but it is inclined to it. For this azimuth, only ion incidence along the line $\eta=0$ was studied. Also here, a strong reduction is seen;

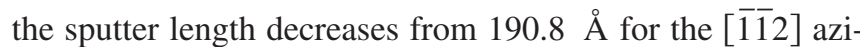




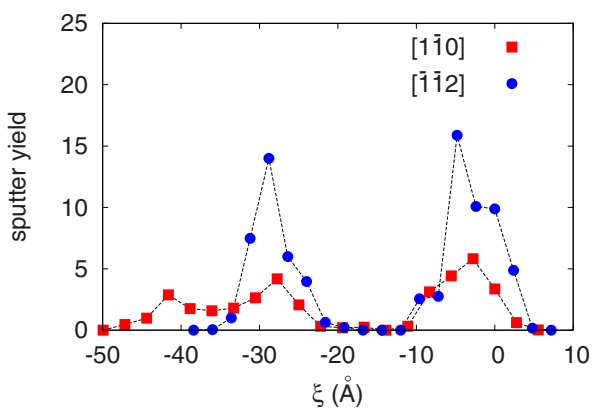

FIG. 7. (Color online) Azimuth dependence of sputtering. Sput-

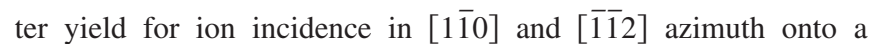
7 -island as a function of $\xi$ for a stripe of width $r_{\mathrm{NN}}$ around $\eta=0$, Eq. (9). Lines to guide the eyes.

muth by $53.5 \%$ to $102 \AA$ for the [1 $1 \overline{10}]$ azimuth. As outlined in detail in Ref. 3, the reason for this decrease lies predominantly in the fact that the (111) terrace appears more corrugated for an ion along the [1 $\overline{10}]$ azimuth; as a consequence the contributions of the indirect-hit zone to sputtering are reduced. Furthermore, along the $[1 \overline{1} 0]$ azimuth, ions view the island (and the surface) as an array of [1 $1 \overline{1} 0]$ axial atomic strings, which makes it easier to avoid collisions; as a consequence the interaction with the ascending edge of the island is reduced and also the direct-hit peak in Fig. 7 decreases.

\section{E. Fate of the island}

As soon as the ion sputters the surface, a sizable amount of energy has been imparted to the island and we may inquire about its fate. From sputtering of step edges it is known that tens of atoms may be torn out of the step edge; the lower terrace widens at the cost of the upper terrace. ${ }^{1}$ Similarly strong effects are observed for our adatom islands.

Figure 8 shows the number of island atoms that have been sputtered. This figure has to be compared to the total number of sputtered atoms shown in Fig. 4. Note that the data shown in Fig. 4 include all sputtered atoms, both substrate and island atoms. A sizable fraction of the sputtered atoms origi-

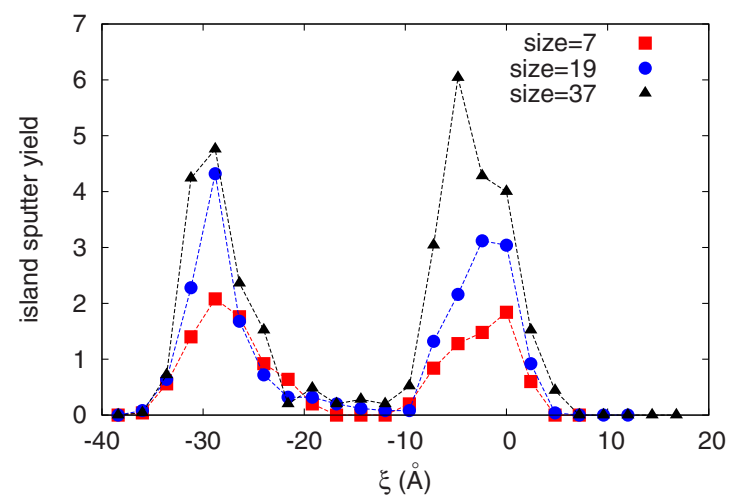

FIG. 8. (Color online) Number of atoms sputtered off the island as a function of the distance $\xi$ to the ascending island edge. Data averaged over ion impact for a stripe of width $r_{\mathrm{NN}}$ around $\eta=0$, Eq. (9). Lines to guide the eyes.

nate from the island; it is $17(26,37) \%$ for the 7- $(19-, 37-)$ island.

We present in Fig. 9 a selection of top views of the 37island after ion impact. The ion trajectories leading to these events are shown in Fig. 10. Due to the strong variation in the ion trajectories with impact point, quite different surface topographies are visible. We note that both for direct and indirect hits we find strong variations in sputter yield and surface topographies. For events of average or larger sputter yield, we find that often the island is destroyed, and also the terrace around it becomes rough. The ion trajectories in these cases often were bent into the substrate beneath the island by the first ion-target atom collision; thus a collision spike could develop under the island which produced abundant sputtering. However, we also find cases of abundant island sputtering, where the ion trajectory only interacts with the island atoms after being reflected (once or twice) from the substrate surface.

Besides the erosion of the island, also a momentum is imparted to the island in the direction of the impinging ion. As a consequence, those island atoms that have not been sputtered will have moved on average "downstream" the ion beam. Figure 11 quantifies this effect of ion-induced mobility by showing the distance by which the island center-of-mass moved. We see that both in the direct- and indirect-hit zone
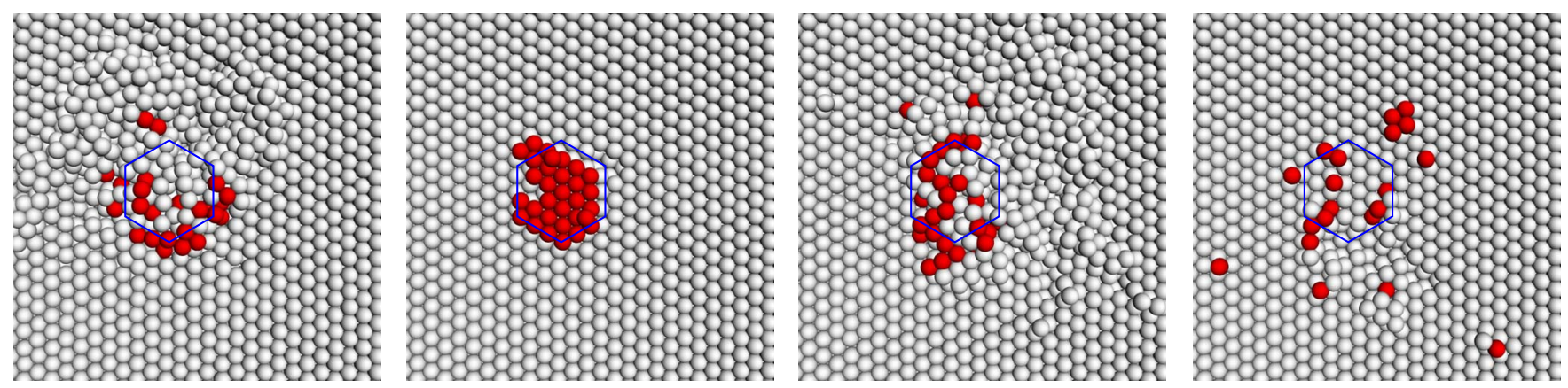

FIG. 9. (Color online) Top views of the surface after various ion impacts on a 37-island. Original island atoms are colored red. The blue hexagon marks the initial boundary of the island. (a) Case of abundant sputtering. $Y=17, Y_{\text {isl }}=7$, and $\xi=-2.8 \AA$, direct hit. (b) Case of average sputtering. $Y=11, Y_{\text {isl }}=5$, and $\xi=-26.9 \AA$, indirect hit. (c) Case of average sputtering: $Y=12, Y_{\text {isl }}=5$, and $\xi=-27.6 \AA$, indirect hit. (d) Case of abundant island sputtering. $Y=17, Y_{\text {isl }}=12$, and $\xi=-27.1 \AA$, indirect hit. 

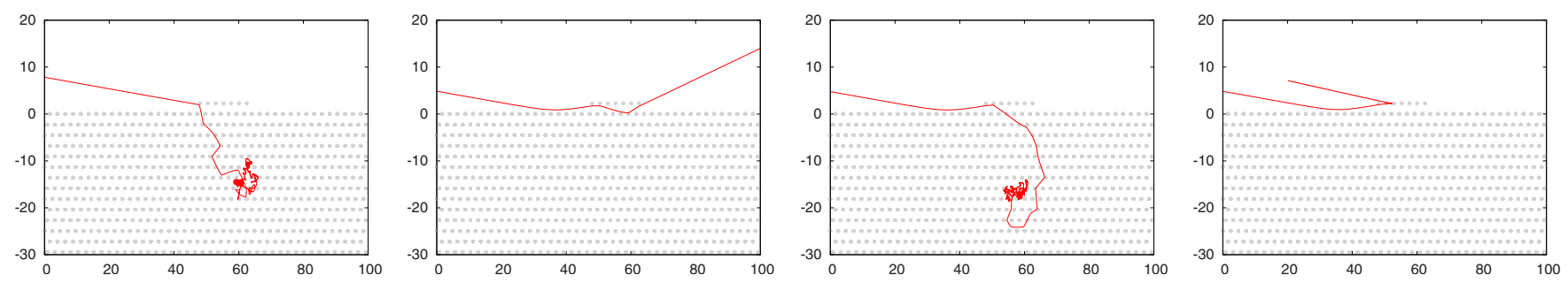

FIG. 10. (Color online) Side views of the ion trajectories (red) of the impacts in Fig. 9. The substrate and island atoms (gray) are shown at their initial positions before impact. The axes denote length scales in angstrom.

an average motion of $1 r_{\mathrm{NN}}$ is possible; this effect remains unweakened up to the largest island size studied by us, $N$ $=37$.

\section{EXPERIMENTAL RESULTS}

\section{A. Experiments}

In order to study the effect of small adatom islands on the erosion rate we performed a dedicated experiment investigating ion erosion of $\mathrm{Pt}(111)$ with adsorbed adislands at grazing incidence.

As a preparative step an amount of $\Theta=0.09 \mathrm{ML}$ of $\mathrm{Pt}$ is deposited on Pt (111) at $155 \mathrm{~K}$ to fabricate small adislands. The corresponding STM topograph of Fig. 12(a) shows a large number of small adatom islands which are mostly of monatomic height, irregular in shape and size. In the STM topograph the deposited amount appears larger than indicated above due to convolution of the STM tip with the adatom island shapes. For coverage calibration the morphology has been annealed for $120 \mathrm{~s}$ to $650 \mathrm{~K}$. Due to diffusion and coarsening much larger compact hexagonal adatom islands result, as shown in Fig. 12(b). Thereby coverage determination with little error is possible.

To obtain the size distribution of the adislands formed at $155 \mathrm{~K}$ we proceeded as follows. The apparent size distribution of adislands was measured with the help of a flooding algorithm. ${ }^{24}$ It was then corrected for tip convolution effects by multiplying with the quotient of calibrated coverage at $650 \mathrm{~K}$ and the apparent coverage at $155 \mathrm{~K}$. The result is shown in Fig. 13 as the probability $f(N)$ that an island consists of $N$ atoms. The average island size is $\bar{N}=50$ atoms with

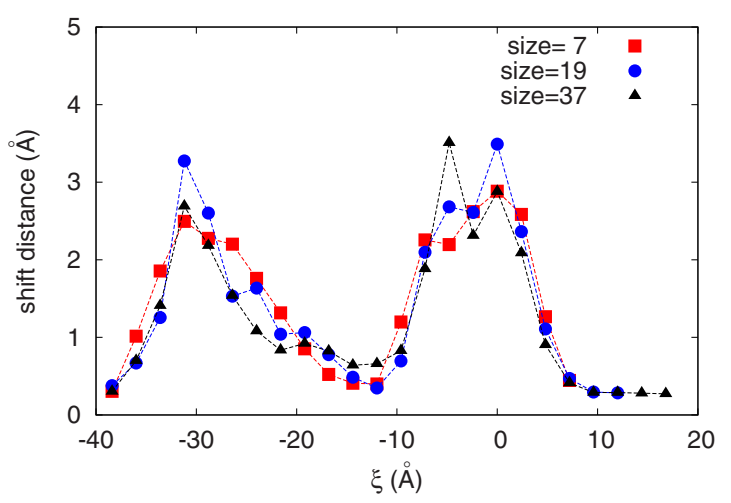

FIG. 11. (Color online) Shift of island after bombardment by a single ion. Data obtained as in Fig. 8. Lines to guide the eyes. a large standard deviation of 42 atoms; due to its skewness the distribution has its maximum at smaller island sizes, in the region of $N=16-27$.

In order to investigate the effect of the adatom islands on the sputtering yield, we chose a surface with adatom islands formed at $155 \mathrm{~K}$ through deposition of $0.09 \mathrm{ML} \mathrm{Pt}$ atoms and exposed it to a fluence of 0.5 MLE $5 \mathrm{keV} \mathrm{Ar}^{+}$ions at $\vartheta$ $=83^{\circ}$ along the $[1 \overline{1} 0]$ azimuth at $155 \mathrm{~K}$. The fluence was chosen small enough to ensure a removed amount well below $1 \mathrm{ML}$ but large enough to limit the errors in coverage analysis. The resulting morphology is shown in Fig. 14(a). Due to the ion exposure, the surface morphology has changed significantly. In addition to the large number of adatom islands the surface displays now a large amount of branched vacancy islands. In order to quantify the amount of removed material in Fig. 14(a) the sample has been heated up to $720 \mathrm{~K}$ for $120 \mathrm{~s}$ and subsequently imaged at room temperature [see Fig. 14(b)]. The annealing step heals all subsurface damage (especially bulk vacancies) to the surface and thereby ensures proper coverage determination. ${ }^{25,26}$ In addition, errors due to tip effects are minimized by the large size of the vacancy islands. The area of vacancy islands of monolayer depth after annealing is $0.25 \mathrm{ML}$. Taking into account that the surface was precovered with 0.09 ML adatom islands before the bombardment, the total amount of removed material equals $\Theta_{\text {rem }}=0.09 \mathrm{ML}+0.25 \mathrm{ML}$ $=0.34 \mathrm{ML}$ with an estimated error of $\pm 0.02 \mathrm{ML}$.

\section{B. Analysis}

The average erosion rate $\bar{Y}$ of $\mathrm{Pt}(111)$ in the presence of adatom islands is simply the amount of removed material $\Theta_{\text {rem }}$ divided by the ion fluence $F$,

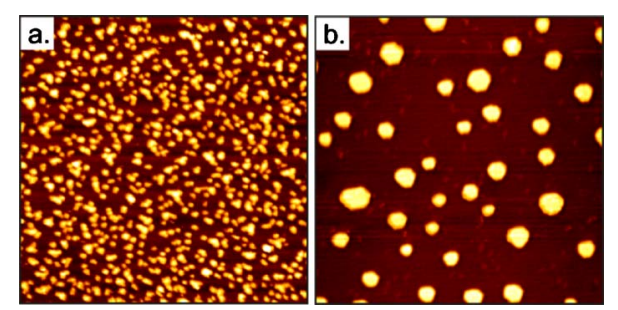

FIG. 12. (Color online) (a) STM topograph after deposition of 0.09 ML Pt on Pt(111) at 155 K. (b) Same experiment as in (a) but after subsequent annealing to $650 \mathrm{~K}$ for $120 \mathrm{~s}$. Image size is $850 \AA \times 850 \AA$. 


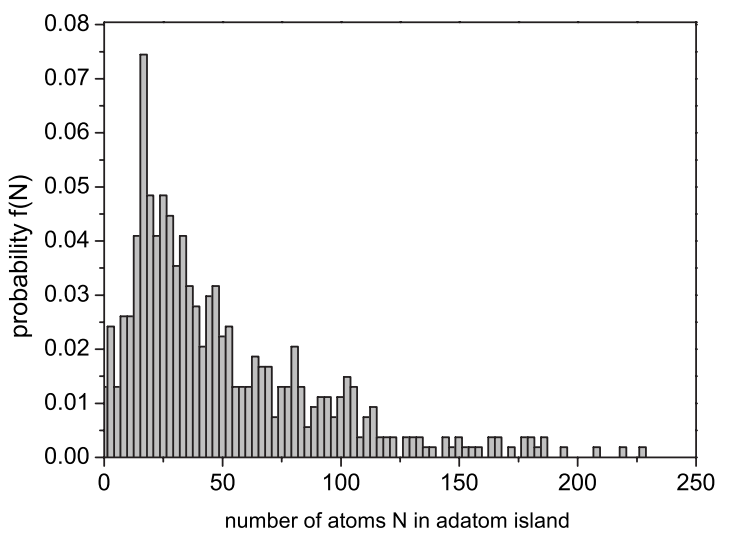

FIG. 13. Normalized size distribution of Pt islands formed after deposition of 0.09 ML Pt at $155 \mathrm{~K}$.

$$
\bar{Y}=\frac{\Theta_{\mathrm{rem}}}{F}=\frac{0.34 \mathrm{ML}}{0.5 \mathrm{MLE}}=0.68 .
$$

When comparing this number to the result of the molecular-dynamics simulations, some care is necessary. In the simulation, after every impact on an adatom island the simulation is reset. In experiment, after an ion has hit an adatom island, the experiment is not reset, but additional impacts hit the modified adatom island. During continued sputtering also vacancies are created, adatoms are sputtered and adatoms are pushed onto the surface layer due to impacts. Therefore our erosion rate represents only to a limited extent the erosion rate of the initial state. However, this unfavorable situation is hard to overcome in a real experiment. Fortunately, with the chosen experimental parameters the density $\rho$ of illuminated island steps (density of ascending steps along a line in the direction of the ion beam) remains almost constant during ion exposure. Prior to exposure it is $\rho=0.019 \pm 0.001 / \AA$ [Fig. 12(a)] and after ion exposure $\rho$ $=0.016 \pm 0.002 / \AA$ [Fig. 14(a)]. Therefore the surface is for the small ion fluence used close to a steady state of adatom island production and annihilation and the resulting $\bar{Y}$ may be considered characteristic for the initial adatom island concentration; still it represents only an estimate.

As the density of ascending adatom island steps stays roughly constant during erosion, we may use the formalism introduced in Ref. 3 to obtain the average yield of an adatom island step $Y_{\text {step }}$. It is

$$
Y_{\text {step }}=\frac{\bar{Y}}{\rho x_{c}} .
$$

From this equation we deduce a step edge sputtering yield of $Y_{\text {step }}=0.98$.

It is meaningful to bring the numbers measured here into the context of previous ion beam erosion experiments of $\mathrm{Pt}$ (111) performed with identical ion beam parameters. The erosion rate of $\bar{Y}=0.68$ measured here is significantly larger than the sputtering yield of the flat terrace for which we found $Y_{\text {terrace }}=0.08 .^{2}$ This difference signifies that adatom islands exhibit a major effect on sputtering under grazing incidence conditions. For our adatom islands with an initial

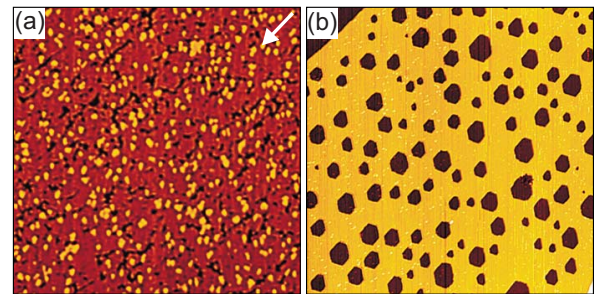

FIG. 14. (Color online) (Color online). (a) STM image after the adatom covered surface in Fig. 12(a) has been exposed to 0.5 MLE $5 \mathrm{keV} \mathrm{Ar}^{+}$at $\vartheta=83^{\circ}$ and $155 \mathrm{~K}$. The arrow indicates the direction of the ion beam. Image size $850 \AA \times 850 \AA$. (b) Same experiment as in (a) but after subsequent annealing to $720 \mathrm{~K}$ for $120 \mathrm{~s}$. Image size $1750 \AA \times 1750 \AA$

average size of 50 atoms we derive a step edge yield $Y_{\text {step }}$ $=0.98$. This value has to be compared with the step edge yield of extended steps, which under identical ion beam conditions amounts to $Y_{\text {step }}=4.6 .^{3}$ Thus, experimentally we find a step of a small adatom island to be about a factor 4-5 less effective in sputtering than extended steps separating two terraces.

\section{Comparison theory-experiment}

Experiment gave an erosion rate of $\bar{Y}=0.68$, Eq. (15), for an initial surface coverage of $\Theta=0.09$. We can compare with theory by taking the distribution of adisland sizes, $f(N)$, Fig. 13, into account. In analogy to Eq. (5) we may write

$$
\bar{Y}=\Theta \sum_{N} y_{N} f(N) .
$$

In order to evaluate this sum, we use Eq. (14) for $N \geq 20, y_{1}$ for $N \leq 4$, and $y_{7}$ for $5<N<19$; because of the [1] 10$]$ azimuth, the latter sputter coefficients have been reduced by $50 \%$ from their values in Table I, cf. the discussion in Sec. III D. The summation, Eq. (17) then gives $\bar{Y}=0.73$, which is in satisfactory agreement with the experimental value. We note that if we disregard the spread in adisland sizes, and simply use the average value $\bar{N}=50$, we obtain using Eq. (14), $\bar{Y}=\Theta y_{50}=0.63$, which is not far away from the better estimate Eq. (17). This demonstrates that our theoretical value is quite robust; any errors committed in the determination of $y_{N}$ have only mild consequences for determining the average $\bar{Y}$.

The trend noted at the end of Sec. IV B above-that the step of an adisland is less efficient in inducing sputtering than an extended step separating large terraces - shows up in exact analogy in our simulation results. While a flat terrace exhibits zero sputtering, $Y_{\text {terrace }}=0.00,{ }^{2}$ and the sputtering of the ascending step edge of an extended terrace was found in simulation to be $Y_{\text {step }}=5.6,{ }^{3}$ our present result shows that adislands lead to considerably less sputtering, $\bar{Y}=0.73$, than the extended steps-in good agreement with the experimental data mentioned above.

The simulation results have been obtained for $0 \mathrm{~K}$ while the experimental data were measured at $155 \mathrm{~K}$. Ref. 3 shows 
that temperature may have a sensitive influence on sputtering from a step. In that reference it was shown that sputtering from a B step increases by $60 \%$ when temperature is raised from 0 to $550 \mathrm{~K}$. A linear interpolation would hence predict a $17 \%$ effect of temperature for our experimental temperature of $155 \mathrm{~K}$. However, we feel that the influence of temperature on adisland sputtering will be smaller than on step sputtering. For steps, temperature had the effect that channeling trajectories are shortened and contribute stronger to sputtering; this effect is missing for adislands.

We point out that in view of the significant uncertainties involved in the determination of the experimental erosion rate (specifically, the superposition of the effects of many impacts in experiment) the quantitative agreement achieved between experiment and simulation may be fortuitous and partly due to error cancellation. However, the experimentally observed trend that the step of an adisland is less efficient in inducing sputtering than an extended step separating large terraces confirms our simulation results at least in a qualitative manner. Future simulations might study the effect of a higher-fluence irradiation on an adisland-covered surface. We refrain from such an investigation here, as we think that the study of a well-characterized surface, containing a single adisland of known size, constitutes an appropriate starting point for analyzing the effect of adislands on sputtering.

\section{CONCLUSIONS}

Sputtering by ions at grazing incidence angles is controlled by the atomic roughness of the flat surface. Besides surface steps, adatom (or vacancy) islands will be present on surfaces and induce sputtering. Since only the ascending step edges of islands, but not island atoms on the terrace behind the ascending step, are active for sputtering, we predict that sputtering decreases with the number $N$ of island atoms as $1 / \sqrt{N}$. Our simulations show that islands containing 10-20 atoms are most effective for sputtering; smaller islands, and in particular adatoms are less effective.

Qualitatively, the effect of single adatoms, extended islands and steps is similar: both direct hits of the ion on the ascending step edge and indirect hits on the terrace in front of the step, such that the reflected ion hits the step edge, contribute. Quantitatively, the differences between the different defects originate mainly from the contribution of the indirect hits. For the single adatom, direct hits dominate while indirect hits contribute only $20 \%$ to sputtering; for a B-step indirect hits contribute up to $50 \%$. As a consequence, for small islands, $N \lesssim 10$, the sputter yield drops slightly. For a step, in addition ions which have been subsurface-channelled contribute. In total, for constant length of the exposed ascending step, large islands are most effective in sputtering. A dedicated experiment performed by us with adatom islands in the size region of $N \cong 50$ demonstrates that adislands induce less sputtering per exposed step length than extended terrace steps; the measured data agree reasonably well with our simulations.

In a simple model, the total sputter cross section can be estimated to be the product of the sputtering for central impact and the exposed ascending step width, Eq. (10). Since the exposed step width grows only $\propto \sqrt{N}$, large islands are less efficient for sputtering than small islands-for constant coverage. This is quantified in the sputter coefficient $y_{N}, \mathrm{Eq}$. (13), which gives the sputtering for constant coverage. For $N \gtrsim 20, y_{N} \propto 1 / \sqrt{N}$. For smaller $N, y_{N}$ goes through a maximum, cf. the discussion above.

\section{ACKNOWLEDGMENT}

This work has been supported by the Deutsche Forschungsgemeinschaft through the project Atomic scale surface damage by ion bombardment at grazing incidence. *urbassek@rhrk.uni-kl.de; http://www.physik.uni-kl.de/urbassek/

${ }^{1}$ A. Friedrich and H. M. Urbassek, Surf. Sci. 547, 315 (2003).

${ }^{2}$ H. Hansen, C. Polop, T. Michely, A. Friedrich, and H. M. Urbassek, Phys. Rev. Lett. 92, 246106 (2004).

${ }^{3}$ A. Redinger, Y. Rosandi, H. M. Urbassek, and T. Michely, Phys. Rev. B 77, 195436 (2008).

${ }^{4}$ Y. Rosandi, A. Redinger, T. Michely, and H. M. Urbassek, Nucl. Instrum. Methods Phys. Res. B 267, 2769 (2009).

${ }^{5}$ Y. Rosandi and H. M. Urbassek, Surf. Sci. 600, 1260 (2006).

${ }^{6}$ Y. Rosandi, A. Redinger, T. Michely, and H. M. Urbassek, Surf. Sci. 603, 320 (2009).

${ }^{7}$ A. Redinger, Y. Rosandi, H. M. Urbassek, and T. Michely, New J. Phys. 11, 063011 (2009).

${ }^{8}$ T. Michely and J. Krug, Islands, Mounds, and Atoms, Springer Series in Surface Science Vol. 42 (Springer, Berlin, 2004).

${ }^{9}$ A. Redinger, H. Hansen, U. Linke, Y. Rosandi, H. M. Urbassek, and T. Michely, Phys. Rev. Lett. 96, 106103 (2006).

${ }^{10}$ H. Hansen, A. Redinger, S. Messlinger, G. Stoian, Y. Rosandi, H. M. Urbassek, U. Linke, and T. Michely, Phys. Rev. B 73,
235414 (2006)

${ }^{11}$ S. H. A. Begemann and A. L. Boers, Surf. Sci. 30, 134 (1972).

${ }^{12}$ A. L. Boers, Surf. Sci. 63, 475 (1977).

${ }^{13}$ H. Niehus and R. Spitzl, Surf. Interface Anal. 17, 287 (1991).

${ }^{14}$ H. H. Brongersma, M. Draxler, M. de Ridder, and P. Bauer, Surf. Sci. Rep. 62, 63 (2007).

${ }^{15}$ A. G. J. de Wit, R. P. N. Bronckers, T. M. Hupkens, and J. M. Fluit, Surf. Sci. 90, 676 (1979).

${ }^{16}$ J. Onsgaard, W. Heiland, and E. Taglauer, Surf. Sci. 99, 112 (1980).

${ }^{17}$ A. J. Algra, E. P. T. M. Suurmeijer, and A. L. Boers, Surf. Sci. 128, 207 (1983).

${ }^{18}$ B. Poelsema, L. K. Verheij, and G. Comsa, Surf. Sci. 148, 117 (1984)

${ }^{19}$ H. Gades and H. M. Urbassek, Phys. Rev. B 50, 11167 (1994).

${ }^{20}$ J. F. Ziegler, J. P. Biersack, and U. Littmark, The Stopping and Range of Ions in Solids (Pergamon, New York, 1985).

${ }^{21}$ Y. Rosandi and H. M. Urbassek, Nucl. Instrum. Methods Phys. Res. B 256, 373 (2007). 
${ }^{22}$ H. F. Winters and E. Taglauer, Phys. Rev. B 35, 2174 (1987).

${ }^{23}$ E. Taglauer, Appl. Phys. A: Mater. Sci. Process. 51, 238 (1990).

${ }^{24}$ I. Horcas, R. Fernández, J. Gómez-Rodríguez, J. Colchero, J. Gómez-Herrero, and A. M. Baro, Rev. Sci. Instrum. 78, 013705
(2007).

${ }^{25}$ T. Michely and C. Teichert, Phys. Rev. B 50, 11156 (1994).

${ }^{26}$ T. Michely and G. Comsa, Nucl. Instrum. Methods Phys. Res. B 82, 207 (1993). 